\title{
Evaluation of Students' Innovation and Entrepreneurship Ability Based on ResNet Network
}

\author{
Fei Wang ${ }^{1}$ and Yalu Ying $\mathbb{D}^{2}$ \\ ${ }^{1}$ Human Resources Office, Hangzhou Vocational and Technical College, Hangzhou, Zhejiang 310018, China \\ ${ }^{2}$ Dean's Office, Hangzhou Vocational and Technical College, Hangzhou, Zhejiang 310018, China \\ Correspondence should be addressed to Yalu Ying; 2004010045@hzvtc.edu.cn
}

Received 28 December 2021; Accepted 25 January 2022; Published 22 February 2022

Academic Editor: Hasan Ali Khattak

Copyright (c) 2022 Fei Wang and Yalu Ying. This is an open access article distributed under the Creative Commons Attribution License, which permits unrestricted use, distribution, and reproduction in any medium, provided the original work is properly cited.

\begin{abstract}
As the country's high-quality talents, college students are an important force in national construction. Evaluating the innovative and entrepreneurial abilities for Chinese students will help promote innovation and entrepreneurship education system and improve the reform of educational system and mechanism of colleges, thereby enhancing the innovation and entrepreneurship abilities of college students and then pushing the country into the ranks of a strong country in human resources and a strong country in talents. This work designs a ResNet-based evaluation method to college innovation and entrepreneurship abilities; the main contributions are as follows. (1) When ResNet performs feature extraction, there are problems of bloated network structure and feature loss. A feature extraction backbone network based on ResNet is proposed. To solve the issue of loss for shallow features in process of feature extraction, a skip architecture is added to fuse the shallow details and spatial information with the deep semantic information. To solve the problem of weak model generalization ability caused by the shallow network, a network stacking strategy is proposed to deepen the network structure. (2) Aiming at the problem that ResNet using single-scale feature prediction cannot effectively utilize multiscale features in the network, a multiscale feature prediction is designed. According to idea of feature pyramid, multiple feature maps with different scales are selected for the improved residual network. It designed a multiscale feature fusion strategy for fusing the selected multiscale feature maps into a feature map and evaluated the innovation and entrepreneurship abilities on the fused feature maps. Finally, comparative experiment proves that the improved feature extraction backbone network and multiscale feature scheme can improve performance accuracy on constructed dataset.
\end{abstract}

\section{Introduction}

There were 8.2 million graduates in 2018, 8.6 million in 2019, and 8.73 million in 2020, according to the Ministry of Education's figures. The limited number of jobs cannot meet the increasing demand for employment, and the employment situation is not optimistic. Based on the increasingly employment situation, the state has proposed many policies to promote employment through entrepreneurship at the strategic level. In 2015, the national document required the implementation of a more active employment policy, combining entrepreneurship and employment and driving employment through entrepreneurship and innovation, which gave birth to new impetus for economic development. In 2015, the State Council once again pointed out the need to implement the entrepreneurial leadership plan for college students to drive employment through entrepreneurship. Innovation and entrepreneurship are the requirements of this era and the current social demand. College students are high-quality talents cultivated by the country and an important manpower in the process of national development. With resources and strong potential entrepreneurial capabilities, it is inevitable for college students to guide students to start businesses. Entrepreneurship is a high-quality way to better grasp the value of life, yet the current work climate cannot match the employment needs of all college students. One method to reduce social pressure on joblessness and enhance the employment landscape is to help college students create their own firms and use entrepreneurship to do it. As part of the national innovation-driven development 
strategy, the country must be able to adapt and lead the new normal of economic development. Entrepreneurship activities at the undergraduate level must be built on innovative and entrepreneurial skills. Therefore, college students must be taught how to be innovative and entrepreneurial, and the topic of college students' creativity and entrepreneurship must be studied [1-5].

Reforming college students' innovation and entrepreneurship education is essential for educational advancement. Its goal is to promote and deepen college students' sense of innovation, boost their entrepreneurial practice ability, and teach college students to become the compound innovative talents needed in the new era and contribute to the growth of the country and society. Innovation and entrepreneurship education should emphasize the development of college students' innovation and entrepreneurship skills, as these skills are critical to the success of student-led innovation and entrepreneurship initiatives. The ability of thinking creatively and logically is critical to the success of innovation and entrepreneurship operations. Restricting innovation and entrepreneurship education's efficacy also affects the effectiveness of innovation and entrepreneurship education as well as its reform. Students' creativity and entrepreneurial capacities must be studied in order to fully implement reforms in innovation and entrepreneurship education [6-10].

Ideological and political education study that examines college students' creativity and entrepreneurship abilities is an important component of both theoretical and practical research. For ideological and political education, it is necessary to study the concept and structure of college students' entrepreneurial potential. Ideological and political education reforms necessitate the integration of innovation and entrepreneurial education with ideological and political education in order to satisfy the actual needs of reform and innovation in college education. College students' overall quality can also be improved by this method. On the other hand, the practical requirements of ideological and political education practice innovation include improving college students' innovation and entrepreneurship ability, accurately grasping the status quo, effectively evaluating the level of college students' innovation and entrepreneurship ability, and proposing practical and feasible strategies for cultivating college students' innovation and entrepreneurship ability. Ideological and political education, as a whole, is in need of theoretical and practical innovation, as well as a boost to discipline development, as a result of analyzing college students' creativity and entrepreneurship capacities [11-15].

An issue that cannot be disregarded in the contemporary period is the development of college students' innovative and entrepreneurial abilities. For the sake of national economic development, educational reform and development, and ideological and political education theory and practice, it is important to study college students' ability of thinking creatively. It is the goal of this project to accurately assess college students' creativity and entrepreneurship potential. ResNet is used to complete the evaluation of students' creativity and entrepreneurial potential, which are different from standard methodologies.
In summary, the contributions are as follows: (1) When the traditional ResNet network is used as the feature extraction backbone network for feature extraction, the deep residual network is not only slow, but also not accurate. This work proposes an improved ResNet as the feature extraction backbone network. (2) Analyze the strategy of ResNet using only the output for the last layer of network as the basis for prediction and design a multiscale feature fusion prediction method. This method can utilize the feature maps to further improve the accuracy of capability evaluation efficiently.

\section{Related Work}

Innovation and entrepreneurship, as the two major sectors of economic activity, had always been complicatedly related. Literature [16] put forward the theory of innovation and entrepreneurship for the first time, taking innovation as the key to economic development. It creatively put forward that economic growth was an important proposition that entrepreneurs promote by establishing a new production function to achieve creative destruction. On this basis, the literature in [17] proposed that the national innovation system is formed by the coevolution of society based on the technology-institution analysis paradigm. And it was concluded that innovation and entrepreneurship were affected by multiple dimensions such as economy, system, and culture, and the evolution of the system was complex and changeable. The United States was the first country to conduct innovation as well as entrepreneurship education; entrepreneurship-related courses were established. The American Innovation and Entrepreneurship Education believed that it was important to educate each student for the free development and provide all-round development of talents to meet social needs, not to achieve employment. The literature in [18] believed that the cultivation of core literacy was the key to entrepreneurial education. It should be coordinated and promoted with the help of the school's highquality entrepreneurial education courses, advanced teaching methods, and perfect evaluation system. The literature in [19] put forward after research that improving teaching methods could strengthen the cultivation of key qualities. By publishing difficult assignments, students are motivated, different concepts were explained, and students were encouraged to debate, cultivate innovative thinking, and use real cases to exercise students' practical ability. The literature in [20] demonstrated the role of university entrepreneurship education in promoting business output and the contribution of college students' entrepreneurial practice activities and participation in the formulation and implementation of regional development strategies to economic recovery. The literature in [21] proposed the fact that entrepreneurship should learn from imitation, learn from experiment, learn from problem solving and opportunities, and learn from mistakes. The literature in [22] believed that entrepreneurship education was through the cultivation of entrepreneurial awareness as well as entrepreneurial spirit. It provided college students with genetic code for future entrepreneurship, surpassing the traditional employmentoriented traditional education. Under the absorption of its 
advanced entrepreneurial philosophy, Babson Business School in the USA cultivated entrepreneurial awareness for college students by advocating innovation and entrepreneurship. It adopted forward-looking curriculum teaching and established practical extended teaching, which had set an example for the world's innovation and entrepreneurship field and had gradually developed into the world's most famous innovation and entrepreneurship institution of higher learning.

The literature in [23] believed that the entrepreneurial spirit of Harvard University implies innovative behavior. Therefore, the experience-oriented model of Harvard University placed great emphasis on shaping and cultivating students including entrepreneurial skills, awareness, and spirit. The literature in [24] started with research on innovation as well as entrepreneurship education in the United Kingdom and believed that the British government initiates various entrepreneurial projects to promote the development of innovation and entrepreneurship by establishing diversified funding sources. The literature in [25] put forward a conceptual model for entrepreneurship education. They believed that entrepreneurship education should be based on educational mission. First, distinguish the types and styles of learners on the basis of exploring the needs of potential entrepreneurs and then determine the teaching goals and methods according to the types of entrepreneurs. Finally, to determine the effect of entrepreneurship education, it could be analyzed from both macro- and microaspects. The literature in [26] believed that an individual's entrepreneurial decision-making was determined by entrepreneurial awareness and entrepreneurial potential. Entrepreneurship awareness was affected by factors such as the national social and cultural environment, education, and teaching methods, and entrepreneurial potential was affected by entrepreneurial education in higher education. The literature in [27] conducted a survey of entrepreneurial education activities in five universities in Sweden and found that most schools paid less attention to entrepreneurial education in personal learning in the classroom environment and paid more attention to entrepreneurial practice. It proposed improvements and reforms based on practical entrepreneurship education. The literature in [28] used pre- and postsurvey methods to empirically analyze the degree to which students could conduct targeted learning based on their entrepreneurial abilities after receiving information on compulsory entrepreneurship courses and put forward a learning model, which could generate learning signals for students in entrepreneurship education. It had positive influence on self-evaluation of entrepreneurial skills. The literature in [29] studied the relationship between business skills acquired by students in secondary and higher education institutions and the relationship between psychological and behavioral factors through a mixed analysis of qualitative and quantitative. It also investigated the gap between entrepreneurial knowledge and entrepreneurial abilities acquired by students and proposed methods for cooperation among stakeholders in entrepreneurial education.

\section{Method}

This work will use the ResNet network to evaluate the innovation and entrepreneurial abilities of college students. Different from the traditional ResNet method, this work will improve the ResNet network from three aspects to further improve accuracy of evaluation for college students' innovation as well as entrepreneurship capabilities.

3.1. Backbone Based on ResNet34. This work uses ResNet34, which has a relatively small amount of network redundancy and a relatively simple structure, as the basic backbone network. Figure 1 shows structure of backbone network with ResNet34. This work calls this backbone as ResIE34.

The cube in figure represents feature map; arrows represent the operations used on the feature map. The specific settings of each operation are illustrated in Table 1.

The residual block that composes ResNet34 is stacked by two convolution kernels with a size of $3 \times 3$. After each convolution is completed, BN normalization and ReLU activation function processing are used. The output value of the residual block is equal to the sum of the input value of the residual block and the output value after the second convolution. Residual-1, Residual-2, Residual-3, and Residual-4 in Table 1 are all stacked by multiple residual blocks. The entire network structure is complex, and the network can be divided into two processes in general: the downsampling process performed by ResNet34 and the upsampling process of feature map expansion.

The downsampling process is divided into four steps. The first step is to uniformly scale input picture size to $512 \times 512 \times 3$. In the second step, a convolution kernel with $7 \times 7$ is utilized in convolution layer to perform a convolution operation with a step size of 2 on the input image. The length and width of the output feature map are reduced to $1 / 2$ of the original, and the number of output channels is 64 . In the third step, the output of the convolutional layer 1 will be input to the maximum pooling layer, using a $3 \times 3$ sampling frame and a max pooling operation with size of 2 . Length and width of the output feature map are reduced to $1 / 2$ of the original, and the number of channels remains unchanged. In the fourth step, the output of the pooling layer will be input to the residual module 1 for feature extraction, and the output feature map has the same size as input. Similarly, Residual-2, Residual-3, and Residual-4 will be used in sequence for feature extraction. The following three feature modules will continuously be downsampled, and the final output feature map size is $16 \times 16 \times 512$.

The upsampling process consists of Upsampling-1, Upsampling-2, and Upsampling-3. The upsampling process enlarges output of ResNet34 network to size required by the prediction module. When the upsampling module transforms the feature map size, it needs to consider the scaling of the feature map length and width and the conversion of the number of channels separately, which requires two steps to implement separately. The first layer of the upsampling module uses convolution to convert feature maps. The second layer of the upsampling module uses microstep 


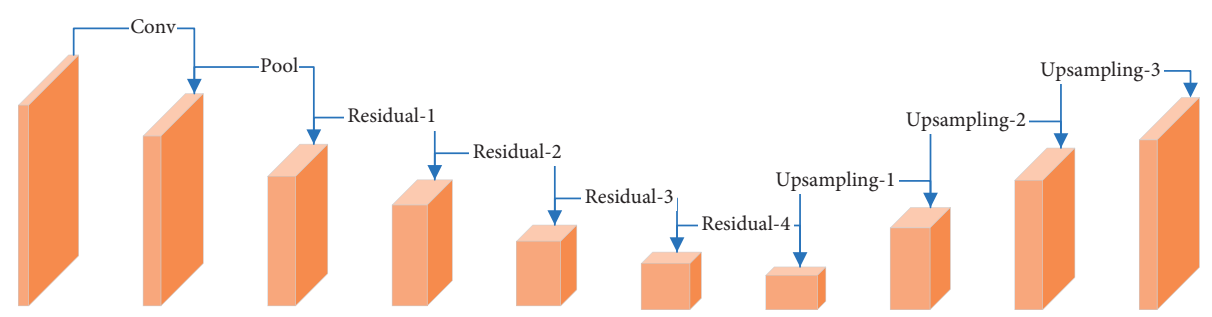

Figure 1: Backbone network structure based on ResNet34.

TABLE 1: ResIE34 network parameters based on ResNet34.

\begin{tabular}{lcc}
\hline Module name & Input feature map size & Output feature map size \\
\hline Conv & $512 \times 512 \times 3$ & $256 \times 256 \times 64$ \\
Pool & $256 \times 256 \times 64$ & $128 \times 128 \times 64$ \\
Residual-1 & $128 \times 128 \times 64$ & $128 \times 128 \times 64$ \\
Residual-2 & $128 \times 128 \times 64$ & $64 \times 64 \times 128$ \\
Residual-3 & $64 \times 64 \times 128$ & $32 \times 32 \times 256$ \\
Residual-4 & $32 \times 32 \times 256$ & $16 \times 16 \times 512$ \\
Upsampling-1 & $16 \times 16 \times 512$ & $32 \times 32 \times 256$ \\
Upsampling-2 & $32 \times 32 \times 256$ & $64 \times 64 \times 128$ \\
Upsampling-2 & $64 \times 64 \times 128$ & $128 \times 128 \times 64$ \\
\hline
\end{tabular}

convolution to enlarge the length and width of the feature map. The upsampling step is divided into three steps. In the first step, output of Residual-1 will be input to Upsampling1 , length and width for feature map will be doubled, and the number of channels will be 256 ; that is, the size of the output feature map will be $32 \times 32 \times 256$. In the second step, Upsampling-2 processes the output of Upsampling-1, and the output feature map size is $64 \times 64 \times 128$. In the third step, Upsampling-3 processes the output of Upsampling-2, and size of output is $128 \times 128 \times 64$, which is the same size as the head network.

\subsection{Embed Skip Architecture to Reduce Feature Loss}

3.2.1. Feature Loss Analysis during Feature Extraction. ResNet34 feature extraction backbone network is an asymmetric hourglass network structure. In the forward derivation process, ResNet34 is first used for downsampling. At this time, the total amount of features carried by the feature map is continuously reduced. Then, use multiple upsampling modules to expand size of feature map; at this time, the total number of features continues to increase. The total feature output of the feature processing module Residual-4 is only $1 / 32$ of the total feature output of the convolutional layer. There are two main reasons for the substantial reduction in the amount of information. (1) The pooling layer uses maximum pooling operations. The sampling box will slide with a fixed step on the input feature map, and finally the elements in the sampling box will be compared. Only the maximum value in the selected box will be selected as the output, and other values will be directly ignored. (2) Second comes the use of convolution operations. The essence of convolution is the filtering effect of the convolution kernel on the image or feature map. Filtering can remove most of the information in the image and emphasize some specific information. The features extracted by shallow convolution are relatively simple, like edges, corners, textures, geometric shapes, and surfaces, while the features from convolution layers are more complex. Many feature pieces of information will be lost in process of extracting both deep and shallow features.

Upsampling module is a process of using microstep convolution to amplify size of feature map; the total number of features will continue to increase. The calculation process is divided into three steps. First, insert a value of 0 in the feature map to increase size of input. Then, calculate the settings for the conventional convolution. Finally, the set conventional convolution is used to convolve the amplified feature map, and the output result is the output of the microstep convolution. After microstep convolution processing, size of feature map increases, and the total number of features increases. The essence of amplification is achieved by inserting 0 values into the feature map, which cannot allow the network to learn more features and make up for the lost features.

In summary, the total number of features is continuously reduced during the downsampling process of the ResNet34 module and will cause a large amount of shallow information to be lost. Although upsampling is a process of increasing the total amount of information, it cannot compensate for the loss of information. The final output features only contain deep information. In the evaluation task of innovation and entrepreneurship capability, different types of identification require different feature information. The shallow features have important reference significance for the prediction task, and the loss of features will cause the decline of prediction accuracy. The feature loss problem can be solved by adding shallow feature information to the upsampling module for network learning.

Skip architecture is proposed by a fully convolutional neural network, whose purpose is making up for shallow information lost in the downsampling process and make the results of semantic segmentation more refined. Its function is to fuse deep, rough, and semantic information with shallow, fine, and surface information to make up for the defects of the loss of shallow features, thereby improving the learning ability of the network. Neural network-based evaluation of innovation and entrepreneurship ability and image segmentation are two different fields. The similarity is that they both need to use a backbone network to extract features from input data. The skip structure is proposed to solve issue of feature information loss in process of feature extraction, which is the same as the feature loss problem in the ResIE34 network. Therefore, the skip architecture is used 
to improve the ResIE34 network to solve the feature loss problem and improve the performance.

3.2.2. Achievement of Skip Architecture. Suppose that the feature map selected during the downsampling process is $a_{1}$ and the output feature map corresponding to the selected upsampling process is $a_{2}$. The output result of feature fusion is $b$, and the general expression for forward derivation of the fusion module is

$$
b=a_{1} \oplus a_{2}
$$

where $\oplus$ is the addition of the corresponding elements of the feature map as

$$
S=\sum_{i=0}^{C} \sum_{j=0}^{H} \sum_{k=0}^{W}\left(A_{i, j, k}+B_{i, j, k}\right),
$$

where $C, W$, and $H$ represent channel number, length, and width.

Suppose that the feature map selected in the sampling process is $a$ and the hidden layer in the dotted frame of the skip architecture is function $f$, so output result corresponding to hidden layer is $f(a)$, and the final feature fusion result is $H(a)$. Therefore, formula (1) can be rewritten as

$$
H(a)=f(a) \oplus a .
$$

Therefore, the skip architecture can be regarded as a special residual structure, and the intermediate network is replaced by a shallow convolutional neural network with a more complex hourglass network structure. According to derivative chain rule, gradient of loss $E$ with respect to the input $a$ is

$$
\frac{\partial E}{\partial a}=\frac{\partial E}{\partial H} \times \frac{\partial H}{\partial a} .
$$

It can be further obtained as follows:

$$
\frac{\partial E}{\partial a}=\frac{\partial E}{\partial H} \times\left(1 \oplus \frac{\partial f(a)}{\partial a}\right) .
$$

Through the analysis of the two processes of forward derivation and backward error propagation of the skip structure, it is found that the feature information can be directly transferred from the shallow layer to the deep layer to compensate for loss of features in downsampling. When network is updating parameters, the gradient can be smoothly transferred back to shallow layer, and the problem of the disappearance of the gradient during the learning process has also been optimized. Therefore, the skip structure can effectively enhance feature extraction and learning ability of ResIE34.

There are two steps to realize the skip structure. First, the fusion location is selected, and the selection rule is to select feature maps with the same size output from the downsampling module and the output size from the upsampling module. Analyze the ResIE34 network structure and select two sets of feature maps that need to be merged. The first group is Residual-2 and Upsampling-2, and the output size is
$64 \times 64 \times 128$ feature maps. The second group is Residual-3 and Upsampling-1, and the output size is $32 \times 32 \times 256$ feature maps. Then, the feature fusion is performed. In the process of forward overturning of the network, the feature extraction of the ResNet34 module will be performed first, and the feature maps generated by Residual-2 and Residual3 will be stored in variables. In the upsampling process, the output result of Upsampling-1 and the output result of Residual-3 stored in the variable are added with corresponding elements to realize feature fusion, and the fusion result is used as the input of Upsampling-2. In the same way, the output result of Upsampling-2 and the output result of Residual-2 are feature-fused. The fused feature map continues to be input to Upsampling-3, and the output result of Upsampling-3 is the final output of the network, completing the feature extraction process of the entire network. Rename the ResIE34 network with the skip structure to ResIES34.

\subsection{Stack Neural Networks to Deepen Network Structure}

3.3.1. Deep Network Optimization Analysis. The performance of a neural network is closely related to size of network, and size of network is determined by depth and width of network. Increasing the width allows each layer of the network to learn more detailed and rich features; increasing the depth can enhance the network's nonlinear expression ability and learn more complex transformations to fit more complex features. The current mainstream increase in the width and depth of the network can improve performance.

Inception V1 [30] is a sparse network structure shown in Figure 2 designed by Google, with the purpose of increasing the width of the network. While widening the network scale, it effectively reduces the calculation parameters and improves the network performance. The network later introduced InceptionV2, InceptionV3 [31], and InceptionV4 [32], which are all improvements based on the idea of widening the network on the basis of InceptionV1.

The deep converged network realizes the improvement of network performance by increasing the horizontal feature fusion of the network, which is also a representative of the widening of the network. VGGNet is a classic example of improving performance by deepening the number of network layers. The residual network adds a residual structure on the basis of VGGNet, which solves the issue of gradient dissipation when network is too deep. The stacked hourglass network uses network stacking to deepen the number of network layers.

Analyzing the method of increasing depth and width, it is found that the method of increasing the width of the network requires changes in the underlying structure of the network and cannot be applied to the ResIES34 network. And the computational cost of increasing the width of the network is higher than increasing the depth, which is the reason why the current neural network continues to deepen the network depth and the network width increases less. ResIES34 has a relatively shallow network layer and needs to increase the network depth to improve performance. 


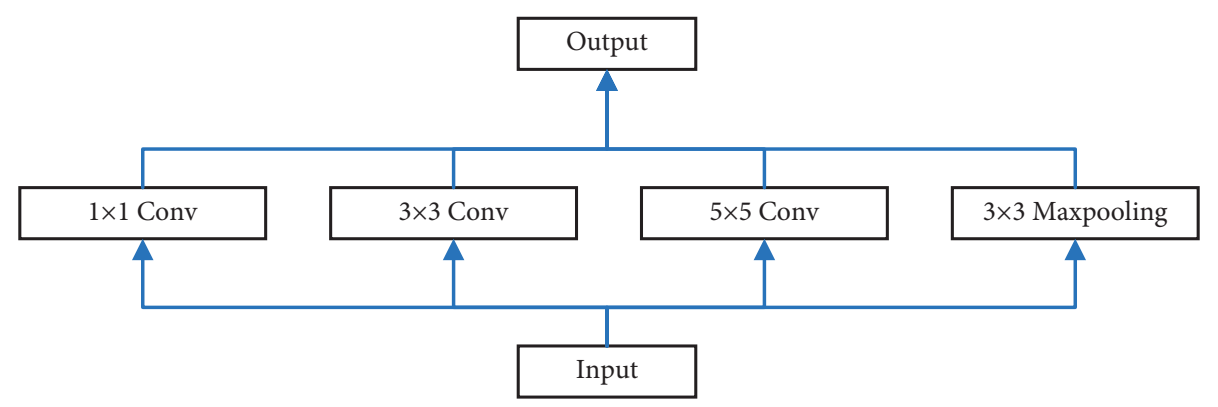

FIgURE 2: Inception structure.

Analysis of the residual network performance at different depths shows that the ResNet50 and ResNet101 networks are constantly deepening, but the improvement in performance is not obvious. Therefore, the method of using network stacking is proposed to increase the network depth. This method regards ResIES34 as a network module as a whole and uses a stacking method to increase depth of neural network. At the same time, each network module contains a skip architecture, which can deepen the neural network and enhance the feature extraction ability, while greatly reducing the feature loss caused during the feature extraction process.

3.3.2. Stacked Network Implementation. There are two difficulties when stacking multiple neural networks. The first is how to connect multiple networks. The second is how to determine the network scale and select the appropriate number of stacked networks. After analyzing the ResIES34 network structure, a new type of network cascading method is proposed. The first network is ResIES34. The network used for subsequent stacking is a feature extraction module formed after removing the convolutional layer, pooling layer, and the first residual module on the basis of ResIES34. This module is an hourglass network structure with the input and output feature map sizes being $128 \times 128 \times 64$, and then the module is named HGNet. The output size of the ResIES34 network is $128 \times 128 \times 64$. When connecting to the HGNet network, there is no need to add a feature conversion module. The direct cascade method can be used; the output of previous network is input of next network. The advantages of this improved method are as follows. The first pass uses the method of direct stacking of the network and does not need to introduce a new conversion module, thereby improving the operating efficiency of the network. The second is that this cascading method can select different network depths according to different needs.

Considering the mutual constraints among factors such as dataset size, network depth, and speed requirements, stacking two neural network modules is proposed as the final feature extraction backbone network. Figure 3 shows the overall structure of the improved feature extraction backbone network based on the residual network. This work calls it as ResIESS34.

The input image first uses the ResIESS34 network for feature extraction, and the output size is $128 \times 128 \times 64$ feature maps. The feature map will be directly input to HGNet for feature extraction, and there is no need to transform the size of the feature map between the two networks. HGNet network is a feature extraction process which first downsamples and then upsamples. The number of downsampling modules and the number of upsampling modules in this process are the same. The final output feature map size is the same as the input size, which is still $128 \times 128 \times 64$.

\subsection{Multiscale Feature Network}

3.4.1. Multiscale Feature Selection. The ResIESS34 network is a complex structure formed by stacking two networks, and many feature maps of different scales will be generated during the network calculation process. How to select feature maps for multiscale processing is a key issue to improve network performance. The second network HGNet stacked by ResIESS34 is compared with the multiscale feature pyramid model.

Bottom-up path: the structure is composed of ResNet_34 after removing the convolutional layer, pooling layer, and Residual-1. After the feature map is input to the network, it will be sequentially downsampled through Residual-2, Residual-3, and Residual4 , and feature maps of three scales are generated. Suppose that the generated feature map is a set $\{A 1, A 2$, $A 3$, where the scales of $F 1, F 2$, and $F 3$ are $64 \times 64 \times 128$, $32 \times 32 \times 256$, and $16 \times 16 \times 512$, respectively. The feature maps of these three sizes are stacked from bottom to top to form a feature pyramid structure.

Top-down path: the structure is composed of an upsampling module formed by a combination of deformable convolution and upsampling convolution. The A3 feature map is sequentially upsampled three times by Upsampling-1, Upsampling-2, and Upsampling-3, so that the output feature map size of the network gradually expands. The output feature map is recorded as a set $\{B 1$, $B 2, B 3\}$, where the feature map sizes of $B 1, B 2$, and $B 3$ are $32 \times 32 \times 256,64 \times 64 \times 128$, and $128 \times 128 \times 64$, respectively. They are stacked from top to bottom and form a characteristic pyramid structure.

Intermediate connection: this structure corresponds to the skip architecture in the network, and the function is to fuse the information of feature map and shallow feature map, so that the feature pyramid carries richer information. 


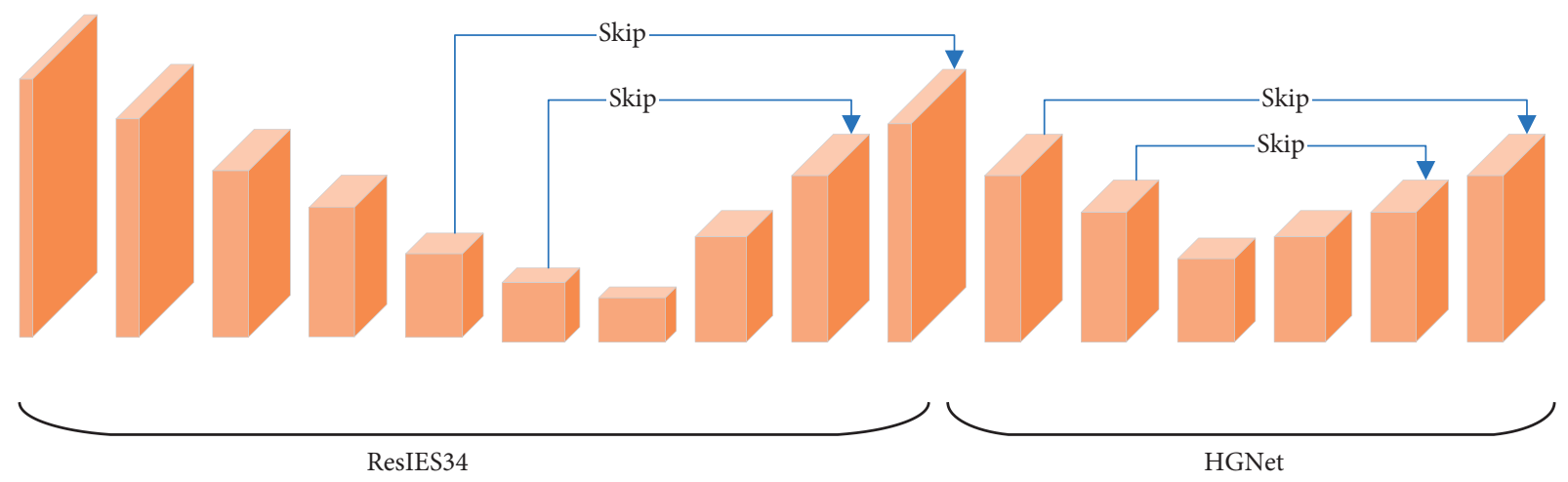

FIgURE 3: The structure of ResIESS34.

The design ideas between HGNet and the multiscale feature pyramid are very compatible. The selection of multiscale feature maps does not need to change the network structure but only needs to follow the design idea of multiscale feature pyramids. It selects the feature map set output by Residual-4, Upsampling-1, Upsampling-2, and Upsampling-3 for multiscale detection. The feature map set selected here is $\{A 3, B 1, B 2, B 3\}$.

3.4.2. Design of Multiscale Feature Fusion Module. In order to fuse feature maps of different scales, a fusion strategy is designed. The input of module is the selected feature pyramid set $\{A 3, B 1, B 2, B 3\}$, and the output size is $128 \times 128 \times 64$ feature maps. The algorithm principle of the multiscale feature fusion module is divided into three steps: unity of the number of feature map channels, unity of the length as well as width for feature map, and the feature fusion. The proposed network is named ResIESSMS34.

The number of feature map channels is uniform. The number of feature map channels in feature pyramid $\{A 3, B 1$, $B 2, B 3\}$ is $512,256,128$, and 64 , respectively, while the number of channels required by the head network is 64 . The method of changing the channel of the feature map is usually implemented using a convolutional neural network. Here, deformable convolution is used to provide a more flexible receptive field for the convolution operation. The size of convolution kernel of deformable convolution is $3 \times 3$, step size is set to 1 , and the convolution process adopts the convolution method of pixel filling. After convolution, the $\mathrm{BN}$ normalization layer and the ReLU activation layer are used for processing. The final network output feature map has the same length and width as input. The ratio of the number of feature map channels generated after feature pyramid is converted and is set to $1: 1: 1: 1$; the number of channels in the pyramid is 16 .

Length as well as width of feature map is uniform. The essence of feature map is a multidimensional matrix. According to the characteristics of matrix operations, matrices of different dimensions cannot be combined during calculation. Therefore, after changing the number of channels, it is significant to normalize length and width of feature map in feature pyramid. The length and width dimensions of the feature map in the pyramid are $16 \times 16$,
$32 \times 32,64 \times 64$, and $128 \times 128$, respectively, and the feature map size accepted by the head network is $128 \times 128$. Therefore, the size of feature map in the feature pyramid needs be normalized to $128 \times 128$.

Feature fusion: each layer of the normalized feature pyramid has the same length, width, and number of channels. The final operation is to splice all the feature maps according to the channel dimensions. The length as well as width dimensions remains unchanged, and all feature maps are stacked back and forth according to the channel dimension. The final output is a $128 \times 128 \times 64$ fusion feature map, which is directly sent to the network for innovation and entrepreneurship evaluation.

\section{Experiment and Discussion}

4.1. Dataset. This work uses two self-made datasets to evaluate the innovation and entrepreneurship ability for college students. Two datasets come from universities in two different regions. The datasets are named IEA and IEB. The IEA contains 1793 training samples and 852 test samples. The IEB contains 5602 training samples and 2115 test samples. Each sample contains 24 feature indicators, as shown in Table 2. The score range for each indicator is 1-100. It should be noted that, to adapt to input size of network, this work will copy and fill these features and the final size is $512 \times 512 \times 3$. The output of the network is the evaluation of innovation and entrepreneurship abilities, using a 1-10 point scoring system. To verify effectiveness of network, this article uses precision, recall, and $F 1$ score. During experiment, the training sample size was uniformly scaled to a size of $512 \times 512$, and each training batch contained 32 samples. During the training process, the training set is all trained once as a cycle, and the total number of times is set to 100 times. The initial learning rate is set to 0.0001 , and the learning rate is attenuated at the 50th and 70th times of training, respectively, and the attenuation rate is 0.1 each time.

4.2. Evaluation on Skip Architecture. As mentioned earlier, this work designed a skip architecture to solve the feature loss problem in the feature generation process. To verify 
TABLe 2: Evaluation index of innovation and entrepreneurship ability.

\begin{tabular}{lccc}
\hline Number & Index & Number & Index \\
\hline$X_{1}$ & Full-time teacher & $X_{13}$ & Risk awareness \\
$X_{2}$ & Part-time teacher & $X_{14}$ & Home country ideal \\
$X_{3}$ & Entrepreneurship mentor & $X_{15}$ & Public interest \\
$X_{4}$ & Ideological quality & $X_{16}$ & Values \\
$X_{5}$ & Knowledge and competence & $X_{17}$ & View of effectiveness \\
$X_{6}$ & Psychological quality & $X_{18}$ & Hardship \\
$X_{7}$ & Political consciousness & $X_{19}$ & Brave spirit \\
$X_{8}$ & Rule of law awareness & $X_{20}$ & Pragmatic spirit \\
$X_{9}$ & Autonomy & $X_{21}$ & Win-win spirit \\
$X_{10}$ & Opportunity awareness & $X_{22}$ & Dialectical thinking \\
$X_{11}$ & Innovative mind & $X_{23}$ & Team thinking \\
$X_{12}$ & Sense of competition & $X_{24}$ & Strategic thinking \\
\hline
\end{tabular}
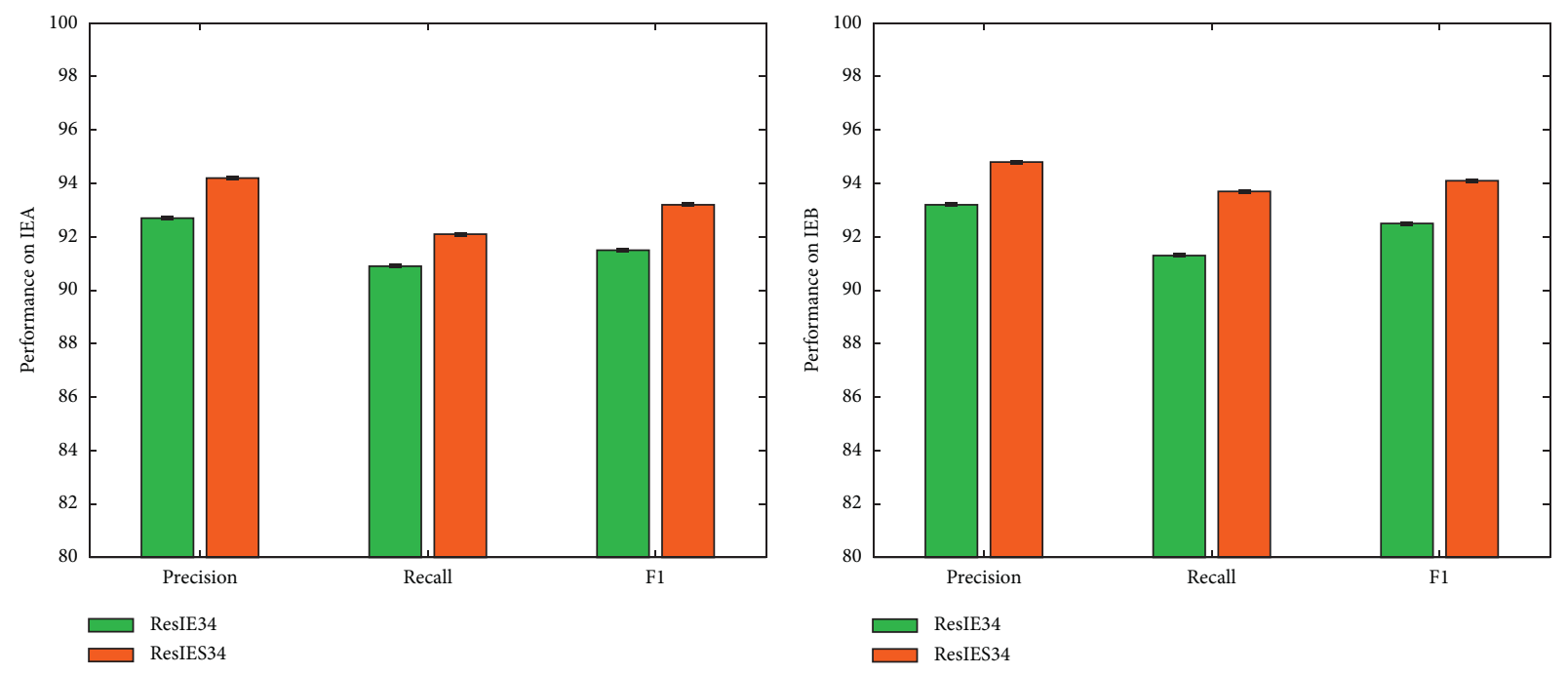

FIgURE 4: Evaluation on skip architecture.

effectiveness of this strategy, a comparative experiment is carried out in this work. The performance of the ResIE34 network without the skip architecture and ResIES34 network with the skip architecture are compared, respectively; the experimental results are illustrated in Figure 4.

Obviously, after embedding the skip architecture, ResIES34 network can obtain $1.5 \%$ precision improvement, $1.2 \%$ recall improvement, and $1.7 \% \mathrm{~F} 1$ score improvement on IEA dataset. At the same time, it can bring $1.6 \%$ precision improvement, $2.4 \%$ recall improvement, and $1.6 \% \mathrm{~F} 1$ score improvement on IEB dataset. The experimental results shown can prove the validity and correctness of the skip architecture designed in this work.

4.3. Evaluation on Network Stack. As mentioned earlier, this work designed a stacked network to learn deeper representation feature. To verify effectiveness of this strategy, a comparative experiment is carried out in this work. The performance of the ResIES34 network without the stacked network and ResIESS34 network with stacked network are compared, respectively; the experimental results are illustrated in Figure 5.
Obviously, after embedding the stacked network, ResIESS34 network can obtain $1.3 \%$ precision improvement, $1.6 \%$ recall improvement, and $1.7 \%$ F1 score improvement on IEA dataset. At the same time, it can bring $1.4 \%$ precision improvement, $1.3 \%$ recall improvement, and 1.4\% F1 score improvement on IEB dataset. The experimental results shown can prove the validity and correctness of the stacked network designed in this work.

4.4. Evaluation on Multiscale Feature. As mentioned earlier, this work designed a multiscale feature fusion module to learn richer information. To verify effectiveness of this strategy, a comparative experiment is carried out in this work. The performance of the ResIESS34 network without the multiscale feature and ResIESSMS34 network with multiscale feature are compared, respectively; the experimental results are illustrated in Figure 6.

Obviously, after embedding the multiscale feature, ResIESSMS34 network can obtain $0.7 \%$ precision improvement, $0.8 \%$ recall improvement, and $0.5 \%$ F1 score improvement on IEA dataset. At the same time, it can bring $0.7 \%$ precision improvement, $0.7 \%$ recall improvement, and 

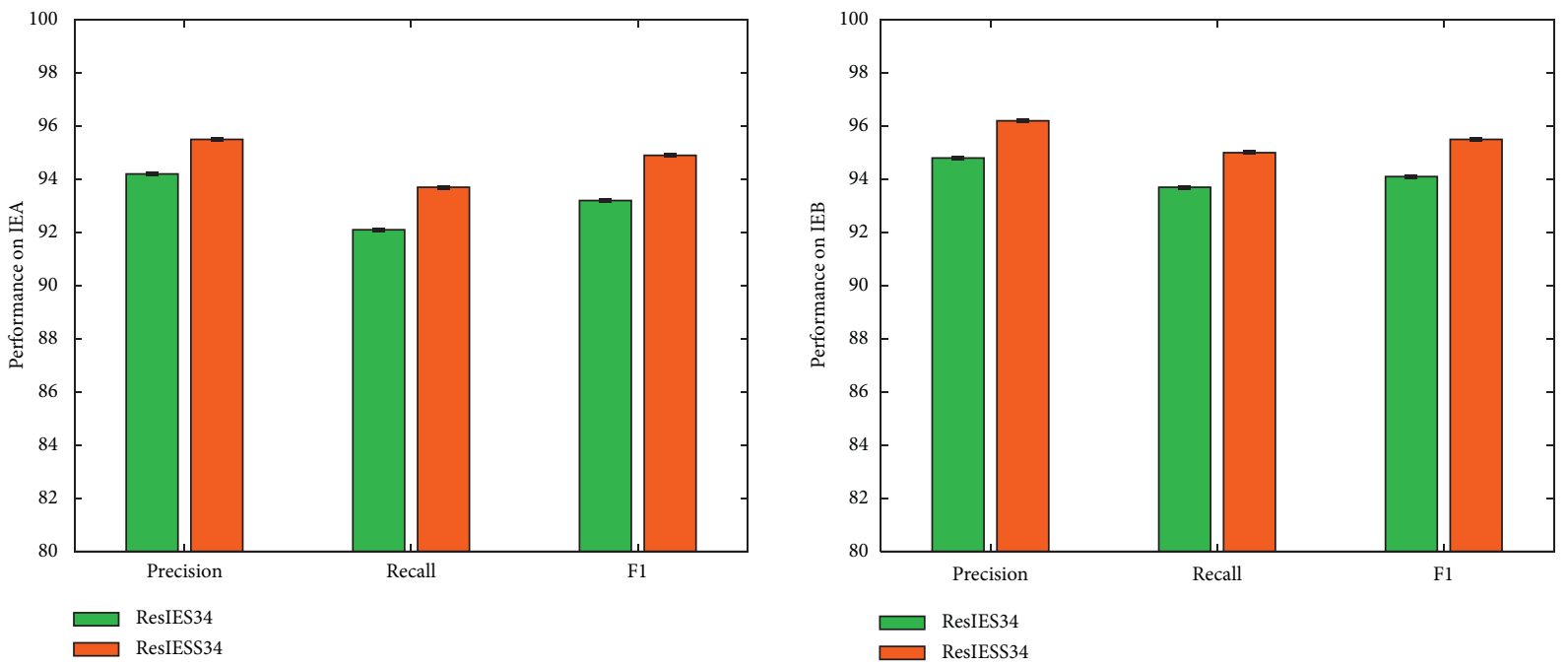

FIGURE 5: Evaluation on network stack.
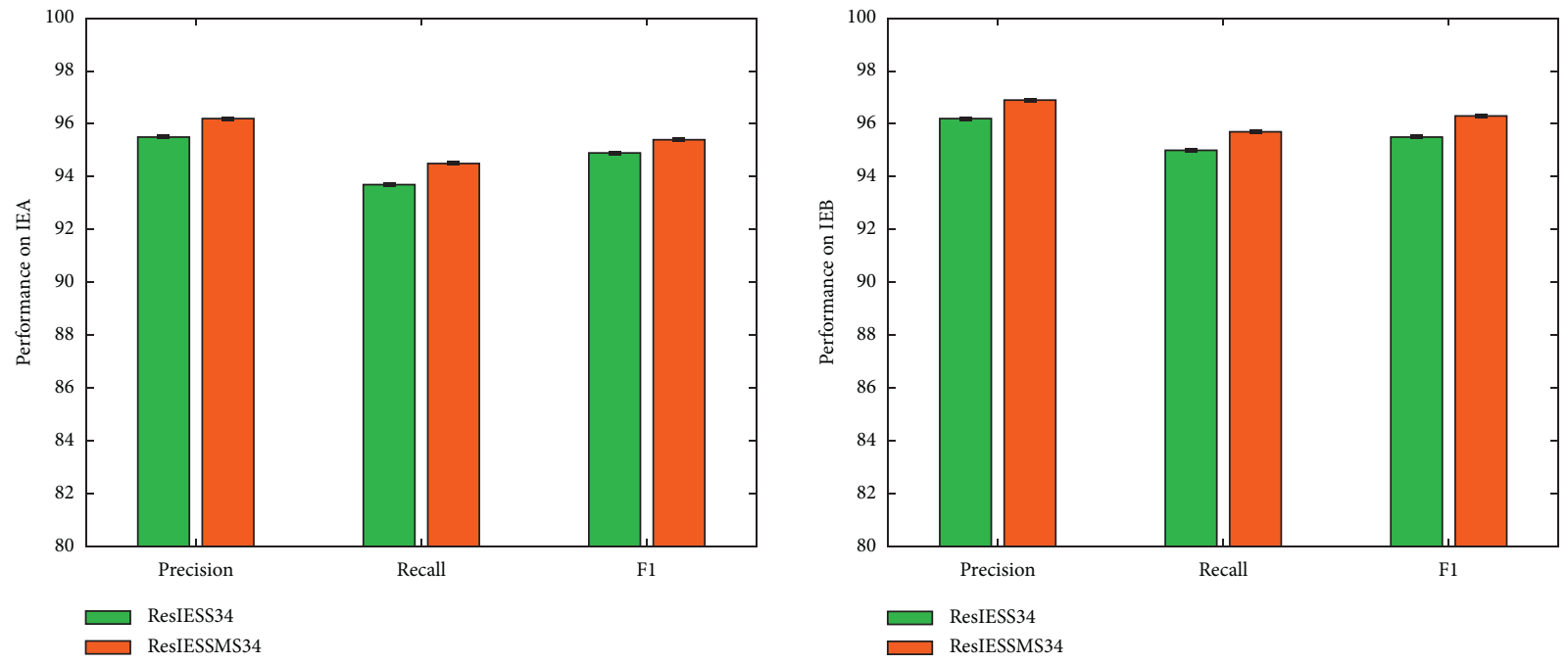

Figure 6: Evaluation on multiscale feature.

$0.8 \%$ F1 score improvement on IEB dataset. The experimental results shown can prove the validity and correctness of the multiscale feature designed in this work.

4.5. Comparison with Other Methods. In order to verify that the network we designed can effectively evaluate the innovation and entrepreneurship abilities of college students, this work compares the designed method with other methods. The methods compared include decision tree (DT), logistic regression (LR), SVM, and BP network. Experimental result is illustrated in Table 3.

It can be seen that, compared with other evaluation methods, the network designed in this work can obtain the best performance. $96.2 \%$ precision, $94.5 \%$ recall, and $95.4 \%$ F1 score can be obtained on IEA. In addition, 96.9\% precision, $95.7 \%$ recall, and $96.3 \%$ F1 score can be obtained on IEB. These illustrated data can verify the effectiveness and correctness of our method.
TABle 3: Comparison with other methods.

\begin{tabular}{lcccccc}
\hline \multirow{2}{*}{ Method } & \multicolumn{3}{c}{ IEA } & \multicolumn{3}{c}{ IEB } \\
& Precision & Recall & $F 1$ & Precision & Recall & $F 1$ \\
\hline DT & 85.1 & 83.4 & 84.7 & 85.5 & 83.7 & 85.2 \\
LR & 88.3 & 86.7 & 87.5 & 89.7 & 87.1 & 88.3 \\
SVM & 91.4 & 89.5 & 90.4 & 92.9 & 91.1 & 92.1 \\
BP & 93.6 & 91.8 & 92.5 & 94.9 & 93.4 & 94.2 \\
Ours & 96.2 & 94.5 & 95.4 & 96.9 & 95.7 & 96.3 \\
\hline
\end{tabular}

\section{Conclusion}

Researching the innovation as well as entrepreneurship abilities for college students and effectively evaluating innovation and entrepreneurship abilities will help promote the innovation and entrepreneurship education system. This is also a necessary condition for a country's technological development. The ability of college students of innovating and starting business is related to future of a country. In this 
paper, the research is on evaluation algorithm of innovation and entrepreneurial ability with ResNet network. Analysis and improvement are carried out from the two directions of feature loss defects and multiscale features. The main research results are as follows. (1) An improved network structure based on ResNet network is proposed. To improve the issue of feature loss in feature extraction process of the network, a skip architecture is added to the network. It is conducive to the direct transfer of shallow features to deep layers and effectively solves the problem of feature loss. To improve generalization ability for network, a network stacking strategy is proposed to deepen network structure. (2) Propose a multiscale feature fusion mechanism, taking into account the fact that the use of single-scale feature maps for evaluation of college students' innovation as well as entrepreneurship capabilities cannot make utilizing multiscale features. For further optimization of the improved deep network based on ResNet network, a new multiscale feature scheme is proposed. First, select a suitable multiscale feature map. Then, input the selected multiscale feature map into multiscale feature fusion module to obtain a feature map containing multiscale information. Finally, the network processes the fusion feature map. The accuracy of the network with multiscale feature fusion has been further improved.

\section{Data Availability}

The datasets used during the current study are available from the corresponding author on reasonable request.

\section{Conflicts of Interest}

The authors declare that there are no conflicts of interest.

\section{Acknowledgments}

This paper was the result of "Research on the Construction Strategy of Entrepreneurial Education Ecosystem in Higher Vocational Colleges," which is the 2021 Chinese Vocational Education Research Project of Zhejiang Province (Project number: ZJCV2021C34).

\section{References}

[1] J. Wang and Y. Tan, "Social factory as prosaic state space: Redefining labour in China's mass innovation/mass entrepreneurship campaign," Environment \& Planning A: Economy and Space, vol. 52, no. 3, pp. 510-531, 2020.

[2] X. Sun, "Exploration and practice of "internet + maker education" university innovative entrepreneurship education model from the perspective of positive psychology," Frontiers in Psychology, vol. 11, p. 891, 2020.

[3] Y. Huang, L. Liu, and L. An, "Are the teachers and students satisfied: sustainable development mode of entrepreneurship education in Chinese universities?" Frontiers in Psychology, vol. 11, p. 1738, 2020.

[4] K. H. Mok and J. Jiang, "Towards corporatized collaborative governance: the multiple networks model and entrepreneurial universities in Hong Kong," Studies in Higher Education, vol. 45, no. 10, pp. 2110-2120, 2020.
[5] G. Lu, Y. Song, and B. Pan, "How university entrepreneurship support affects college students' entrepreneurial intentions: an empirical analysis from China," Sustainability, vol. 13, no. 6, p. 3224, 2021.

[6] S. Zhao, H. Zhang, and J. Wang, "Cognition and system construction of civil engineering innovation and entrepreneurship system in emerging engineering education," Cognitive Systems Research, vol. 52, pp. 1020-1028, 2018.

[7] J. Pei, K. Zhong, J. Li, J. Xu, and X. Wang, "ECNN: evaluating a cluster-neural network model for city innovation capability [J]," Neural Computing \& Applications, pp. 1-13, 2021, https:// doi.org/10.1007/s00521-021-06471-z.

[8] H. Zhang and C. Yang, "The application of big data analysis in the research of innovation and entrepreneurship education for humanities college students[C]," Journal of Physics: Conference Series. IOP Publishing, vol. 1648, no. 2, Article ID 022073, 2020.

[9] L. Ma, Z. Lan, and R. Tan, "Influencing factors of innovation and entrepreneurship education based on the theory of planned behavior," International Journal of Emerging Technologies in Learning (iJET), vol. 15, no. 13, pp. 190-206, 2020.

[10] Y. Wang and B. Tang, "Research and practice on the collaborative education system for the innovation and entrepreneurship of E-commerce major[C]," in Proceedings of the International Conference on Big Data and Informatization Education (ICBDIE), pp. 203-206, IEEE, Zhangjiajie, China, 23-25 April 2020.

[11] X. Qian, H. Shi, C. Ge, H. Fan, X. Zhao, and Y. Liu, "Application research on service innovation and entrepreneurship education in university libraries and archives," International Journal of Computational Science and Engineering, vol. 22, no. 1, pp. 96-106, 2020.

[12] Q. Xiaoxing, "Research on innovation and entrepreneurship education," Higher Education Research, vol. 5, no. 6, p. 209, 2020.

[13] W. Yao, Z. Zhang, and X. Mu, "Research on improvement of innovation and entrepreneurship of university students based on studio cultivation with tutorial system[J]," International Journal of Social Science and Education Research, vol. 3, no. 8, pp. 190-196, 2020.

[14] O. C. Coiado and K. Ahmad, "Introducing first-year medical students to product innovation and entrepreneurship," Medical Science Educator, vol. 30, no. 1, pp. 19-20, 2020.

[15] Y. Shu, S.-J. Ho, and T.-C. Huang, "The development of a sustainability-oriented creativity, innovation, and entrepreneurship education framework: a perspective study," Frontiers in Psychology, vol. 11, p. 1878, 2020.

[16] M. Fritsch, "The theory of economic development - an inquiry into profits, capital, credit, interest, and the business cycle," Regional Studies, vol. 51, no. 4, pp. 654-655, 2017.

[17] C. Freeman, “Technology and economic performance: lessons from Japan[J]," London: Pinter. Freeman Technology and economic performance: Lessons from Japan, vol. 1987, p. 1, 1987.

[18] A. J. Rotherham and D. T. Willingham, "21st-century skills [J]," American Educator, vol. 17, no. 1, pp. 17-20, 2010.

[19] P. Tynjälä and H. L. T. Heikkinen, "Beginning teachers' transition from pre-service education to working life[J]," Zeitschrift für Erziehungswissenschaft, vol. 14, no. 1, pp. 11-33, 2011.

[20] M. J. Escalona, M. Urbieta, G. Rossi, J. A. Garcia-Garcia, and E. R. Luna, "Detecting Web requirements conflicts and inconsistencies under a model-based perspective," Journal of Systems and Software, vol. 86, no. 12, pp. 3024-3038, 2013.

[21] J. Shitundu, "Growth and structure of Tanzanian industrial sector investment and employment[J]," African Journal of Finance and Management, vol. 8, no. 2, pp. 46-45, 2000. 
[22] P. G. Wilhelm, "International validation of the corruption perceptions index: implications for business ethics and entrepreneurship education[J]," Journal of Business Ethics, vol. 35, no. 3, pp. 177-189, 2002.

[23] J. A. Katz, "Fully mature but not fully legitimate: a different perspective on the state of entrepreneurship education," Journal of Small Business Management, vol. 46, no. 4, pp. 550-566, 2008.

[24] S. U. N. Ke, "The British university entrepreneurship education in the 21st century[J]," Comparative Education Review, vol. 10, 2010.

[25] E. Liguori and C. Winkler, "From offline to online: challenges and opportunities for entrepreneurship education following the COVID-19 pandemic," Entrepreneurship Education and Pedagogy, vol. 3, no. 4, pp. 346-351, 2020.

[26] V. Ratten and P. Usmanij, "Entrepreneurship education: time for a change in research direction?" The International Journal of Management Education, vol. 19, no. 1, Article ID 100367, 2021.

[27] E. A. Rasmussen and R. Sørheim, "Action-based entrepreneurship education," Technovation, vol. 26, no. 2, pp. 185-194, 2006.

[28] G. Von Graevenitz, D. Harhoff, and R. Weber, "The effects of entrepreneurship education," Journal of Economic Behavior \& Organization, vol. 76, no. 1, pp. 90-112, 2010.

[29] C. Chiru, L. Tachiciu, and S. G. Ciuchete, "Psychological factors, behavioural variables and acquired competencies in entrepreneurship education," Procedia - Social and Behavioral Sciences, vol. 46, pp. 4010-4015, 2012.

[30] C. Szegedy, W. Liu, Y. Jia et al., "Going deeper with convolutions[C]," in Proceedings of the IEEE Conference on Computer Vision and Pattern Recognition, pp. 1-9, Boston, MA, 7-12 June 2015.

[31] C. Szegedy, V. Vanhoucke, S. Ioffe, J. Shlens, and Z. Wojna, "Rethinking the inception architecture for computer vision [C]," in Proceedings of the IEEE Conference on Computer Vision and Pattern Recognition, pp. 2818-2826, Las Vegas, NV, USA, 27-30 June 2016.

[32] C. Szegedy, S. Ioffe, V. Vanhoucke, and A. A. Alemi, "Inception-v4, inception-resnet and the impact of residual connections on learning[C]," in Proceedings of the AAAI conference on artificial intelligence, pp. 4278-4284, 4 February 2017. 